\section{Games, Groups and the Global Good}

Simon A Levin (ed)

Springer-Verlag GmbH, 2009. 283pp. £108.00, Hardback

ISBN: 978-3540854357

Journal of the Operational Research Society (2013) 64, 1867. doi:10.1057/jors.2013.125

Cooperation-how it comes about, how it is achieved and what its uses and implications are-constitutes an important challenge for the behavioural sciences. This book, an edited volume including contributions from eminent experts on this problem, is an authoritative reading on our present understanding of these issues. A particularly appealing feature of this book is the combination of breadth, in the number of different perspectives it manages to incorporate, with depth, in that even brief chapters are written in a way that provides valuable insight.

The book is organized by three major themes. It starts at the individual level by asking about the evolution of cooperation in a setting where there are self-interested actors. The second section moves on to issues of group formation and finally there is discussion of problems of the commons. This linear transition from the individual to the social organizes the nice diversity of viewpoints represented along the way.

As this is a book in a series in game theory, there are clearly numerous models that apply this theory. However, there is a significant variety-we find out in the two introductory chapters how some biological phenomena are nicely described by evolutionary game models and how cooperation can arise from the game dynamics. However, this leads up to more nuanced discussions about the realism of some aspects of these models and the roles of reputation and network structure in the

\section{Modeling and Simulation for Analyzing Global Events}

John A Sokolowski and Catherine M Banks John Wiley and Sons, 2009. 205pp., \$110.00, Hardback
ISBN: 978-0470478417

Journal of the Operational Research Society (2013) 64, 1867-1868. doi:10.1057/jors.2013.127

Perhaps surprisingly, modelling and simulation (henceforth M\&S) have been used with lesser extent in social sciences or liberal arts than in fields, such as the natural sciences, business, commerce and government. Now the question is why do we need to model and simulate real-world/contrived events? And if we do need it, how do we go about developing theoretical evolution of cooperation. Similarly, even at the level of social systems, we see the discussion of strategic issues in negotiations and apologizing, showing us how agents could resolve difficult issues of cooperation, which is complemented by the discussion of the importance of mechanisms of trust and team level rather than individual reasoning. Indeed, the book covers even more ground than these modelling issues, especially in the second section, by taking on philosophical issues around the evolution and uses of moral systems, even taking on the role and evolution of religion. A final dimension of variety is defined by the combination of theoretical discussions of models, such as regarding the structure of matrix games, and empirical discussions such as based on data regarding city-level social behaviour.

Despite all this diversity, which is a virtue in a collection such as this one, there is indeed a common underlying theme. Much of this book addresses how a simple game theoretic viewpoint on coordination must be augmented by institutional mechanisms and individual-level attributes that play an important role in making cooperation possible. From this reviewer's point of view, the potential readership and audience for this book could have been increased by the inclusion - in addition to the discussion of the state of the art and speculations about future directions - of some further tutorial content summarizing this specific theme independently from the expert essays. However, this is only a minor quibble that may be addressed by simply going back and reading specific chapters, and associated references, as the theme begins to clarify in the reader's mind.

In summary, this is a very interesting and authoritative collection of chapters on the broad topic of cooperation, considering the problem at many levels of description and from a number of methodological standpoints. It should appeal to a wide audience including scientists or graduate students who wish to understand social behaviour through the lens of game theoretic modelling.

\section{University of Edinburgh Subramanian Ramamoorthy}

fundamentals and research design that will come to the aid of practitioners or learners with non-technical background? These are the central concerns of this intriguing volume, which provides a comprehensive and insightful overview while illustrating case studies to better understand the highly complex globalized network through the lens of $M \& S$.

It is believed that, in this reviewer's opinion, initiatives made by influential voices such as Herbert A Simon and his learning by doing, plus the emergence of other computational trends such as behavioural modelling, has inspired this work. Following the critique that the complexity of global events is inadequately represented by a dominant qualitative discourse of liberal arts, this book sets out to fill this paucity in analysis techniques, where qualitative analysis and quantitative numerical representation are integrated (referred to as mixed-methods research) to facilitate an understanding of the cumulative impact of seemingly local events on global views. 